\title{
Kind-Philipp-Stiftung für Leukämieforschung 1972-1982
}

\author{
M. HertI
}

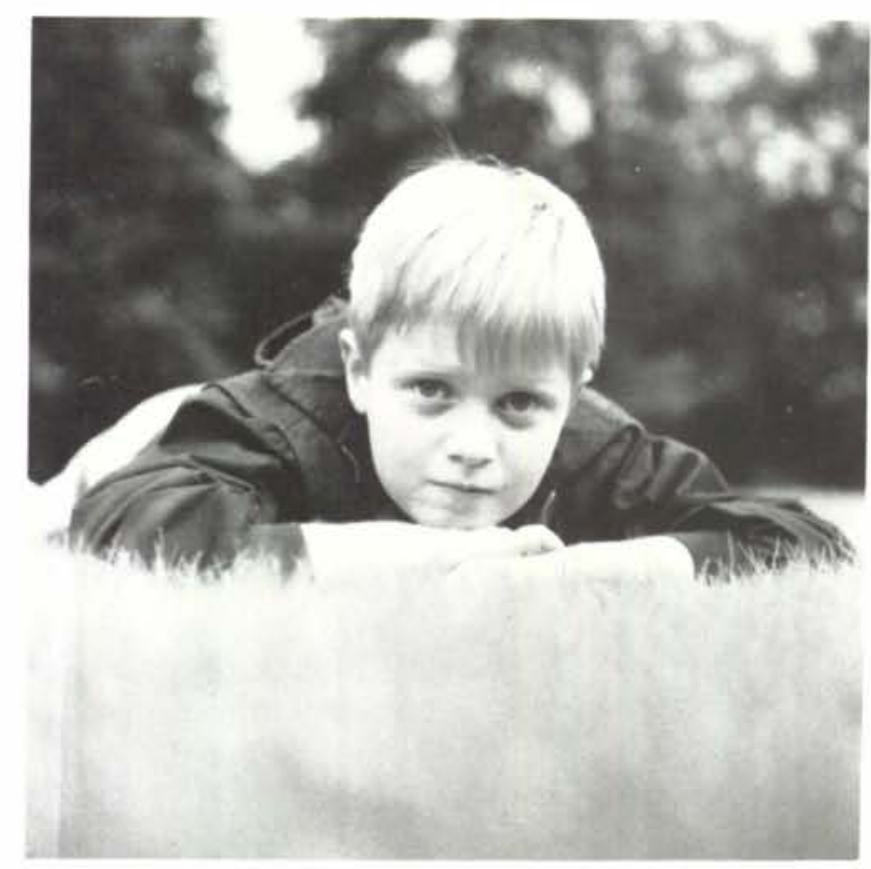

\section{Anlaß für die Stiftung}

Am 2. Mai 1972 verstarb Philipp Reiners, 14 Jahre alt, an einer akuten Leukämie. Die Diagnose war im März 1971 gestellt worden. 4 Wochen später kam Philipp in die Remission. Im November 1971 folgte dann aber der zweite, im Frühjahr 1972 der dritte Krankheitsschub. Philipp wurde nach den damals modernsten Verfahren behandelt, aber alle Hoffnungen waren umsonst. Sein Vater, Dr. Walter Reiners, Inhaber einer Textilmaschinenfabrik in Mönchengladbach, rief eine Stiftung zur Förderung der Leukämieforschung ins Leben, im Gedanken, anderen Kindern und ihren Familien dieses Schicksal in der Zukunft hoffentlich ersparen zu können. Im Stiftungsstatut heißt es: „.. . daß die medizinische Wissenschaft in den Stand gesetzt werden muß, noch vielmehr als bisher in die Grundlagen der Leukämie als Krankheit und daraus in die Möglichkeiten einer nicht nur bessernden, sondern heilenden Therapie einzudringen". Im Herbst 1972 begann die Stiftung, betreut vom Stifterverband für die Deutsche Wissenschaft, ihre Arbeit.

Die Stiftung erhielt auf Vorschlag des behandelnden Arztes den Namen

„,Kind-Philipp-Stiftung für Leukamieforschung“, um neben aller Sachlichkeit und naturwissenschaftlicher Exaktheit, die die Leukämieforschung braucht, darzutun, daß die Krankheitsprobleme in gleicher Größe und Bedeutung auch im Menschlichen liegen und die ärztlichen Bemühungen gleichbedeutend auch in der psychischen Hilfe für ein krankes Kind und seine Eltern bestehen.

\section{Hoffnung auf Heilung}

Als Philipp im Jahre 1971 erkrankte, konnte ein ärztliches Aufklärungsgespräch für die Eltern eines Leukämiekindes praktisch keine reale Hoffnung enthalten. Gewiß, eine Remission zu erzielen, war die Regel, aber Heilungen waren so extrem selten, daß man darauf vernunftigerweise keine Hoffnung setzen konnte. Wieviel hat sich seitdem geändert! Heilungen sind möglich, sie sind eine echte Hoffnung, die den Eltern auf den schweren Weg der Behandlung mitgegeben werden kann. Mit den heutigen Therapieverfahren, die vor allem ein Verdienst von Pinkel und Riehm sind, kann mit einer Heilungsquote von $50-70 \%$ gerechnet werden. Auch die Kind-Philipp-Stiftung hat einen Anteil an dieser ungeahnten günstigen Entwicklung. Diese Zahlen lassen nicht erkennen, unter welchen Mühen und extremen Belastungen diese Wochen und Monate der Behandlung durchgestanden 
werden müssen, oft bis an die existentielle Grenze der Belastungsfähigkeit des Organismus. Solche Zahlen sagen gleichzeitig aber noch etwas anderes aus, was weiterhin der allergrößte Ansporn für eine Verbesserung gegebener Therapieschemata ist: viele, zu viele Kinder können noch nicht gerettet werden. Sie sterben letzten Endes doch, nach Wochen, Monaten oder Jahren, an der Unbeherrschbarkeit der Krankheit oder an den Nebenwirkungen der Therapie.

\section{Geförderte Forschungsprojekte}

Nach dem Willen des Stifters dienen die Mittel einer „Erforschung der Grundlagen der Leukämie, insbesondere des Kindesalters, in diagnostischer und therapeutischer Hinsicht". In den 10 Jahren ihres Bestehens hat die KindPhilipp-Stiftung 42 Forschungsprojekte finanziell unterstützt. Viele Vorhaben verdanken erst dieser Stiftung die Möglichkeit, daß die fruchtbare Arbeit beginnen konnte. So groß die Beträge waren und sind, die der Stiftung zuflossen, so beschränkt waren sie doch auch mit Blick auf die hohen Ausgaben, die eine wissenschaftliche Forschung heute macht. Auf diese Begrenztheit war und ist das Konzept einer Förderung durch die Stiftung abgestimmt. Die Förderungsrichtlinien besagen, daß nur neue Projekte gefördert werden, für die noch kein Kostenträger gegeben ist. Die Förderung ist Starthilfe, ist „Initialzündung“, wie es Walter Reiners immer genannt hat. Und es zeigte sich auch bald, daß für die laufende und erfolgversprechende weitere Arbeit dann auch andere Instanzen eintraten, die Deutsche Forschungsgemeinschaft, die Stiftung Volkswagenwerk, die Deutsche Krebshilfe, um nur die wichtigsten zu nennen.

Im einzelnen wurde auf folgenden Gebieten die Leukämieforschung gefördert:

- Untersuchungen an Leukämiezellen, morphologische, zytochemische, immunologische und andere zellphysiologische Untersuchungen;

- statistische Untersuchungen zur Leukämiehäufigkeit;

- Untersuchungen zur Klassifizierung der malignen Lymphome, die in vielen Fällen Vorstadien der akuten Leukämie sind;

- Therapiestudienreihen;

- Untersuchungen zur Knochenmarktransplantation.

Ein besonderes Anliegen war es, die wissenschaftliche Ausbildung junger Forscher zu fördern und den Nachwuchs mit modernen Forschungstechniken vertraut zu machen. Unter diesen Aspekten wurden die Mittel für zwei Kurse für jeweils 20-30 Hämatologen an der Universität Ulm bereitgestellt.

\section{Kind-Philipp-Expertentagungen}

Ein anderer Gedanke war es, die wissenschaftliche Zusammenarbeit, das freie Gespräch unter den Wissenschaftlern zu fördern. So wurden zwei „Kind-Philipp-Expertentagungen“ organisiert, auf denen Wissenschaftler ihre neuesten Ergebnisse vortrugen und diskutierten. Sie fanden jeweils im Wissenschaftszentrum Bonn-Bad Godesberg statt, 1978 unter der Leitung von Landbeck, 1981 unter der Leitung von Lampert. Diese Tagungen sollen alle zwei Jahre fortgesetzt werden.

\section{Kind-Philipp-Preis für Leukämieforschung}

Aus den Stiftungsmitteln wurde und wird ferner für jedes Kalenderjahr ein Preis für die beste deutschsprachige Arbeit zur Erforschung der Leukämie, insbesondere bei Kindern, ausgeschrieben. Es soll damit - anknüpfend an die hervorragende Tradition der deutschen Hämatologie - der Leukämieforschung ein besonderer Anreiz und eine öffentliche Anerkennung für erfolgreiche Tätigkeit gegeben werden. Der Preis besteht in einer Urkunde und einem Geldbetrag von DM $10000,-$. Er trägt die Bezeichnung „Kind-PhilippPreis für Leukämieforschung“. Ausschreibung und Ubergabe des Preises sind in die Hände des Vorstandes der Deutschen Arbeitsgemeinschaft für Leukämie-Forschung und -Behandlung im Kindesalter e.V. gegeben, eine bewährte, 1966 gegründete hämatologisch-onkologische Arbeitsgemeinschaft aller deutschen Kinderkliniken.

Bisher ist der Preis $7 \mathrm{mal}$ vergeben worden. Folgende Forscher sind mit ihren Arbeiten jeweils auf der Sommertagune der Arbeitsgemeinschaft in Frankfurt ausgezeichnet worden

1972: Dr. Norbert Kluge, cand. med. Almuth Knebel, Dr. Hans Meldris, Dr. Bernd Weichmann, Dr. Gerhard Gaedicke Dr. Shyan Dube und Prof. Dr. Wolfram Ostertag, MaxPlanck-Institut für Experimentelle Medizin, Abteilung Mole kulare Biologie, Göttingen:

„Die Polycythaemia vera als Modell zur Untersuchung der Entstehung von Leukämien".

1973: Prof. Dr. Hansjörg Riehm, Kinderklinik der Freien Universität Berlin:

"Intensive kombinierte Induktionsbehandlung und ZNS. Bestrahlung der akuten lymphoblastischen Leukämie des Kindes - Dokumentation und Bilanz nach drei Jahren".

1975: Prof. Dr. Karl Lennert, Pathologisches Institut der Universität Kiel:

,Klassifikation der Non-Hodgkin-Lymphome, insbesondere der hochgradig malignen Lymphome des Kindesalters".

1976: Dr. Rudolf Eife, Immunologisches Labor der Kinde klinik der Universität München:

„Genuine und iatrogene Immunstörungen bei der akuten lymphoblastischen Leukämie des Kindesalters".

1977: Prof. Dr. Prakash Chandra, Dr. Linda K. Steel, Dr. Horst Laube, Prof. Dr. Bernhard Kornhuber, UniversitätsKinderklinik Frankfurt/M.:

„Molekularbiologische Untersuchungen zur Vinusätiologie eines präleukämischen Syndroms im Kindesalter (Osteomyelofibrose)“.

1980: Dr. Günter Henze und Dr. Hans-Joachim Langermann, Kinderklinik der Freien Universität Berlin:

„Die Prognose der akuten lymphoblastischen Leukämie im Kindesalter in Abhängigkeit von Initialbefunden und Therapie. Eine Analyse von drei Therapiestudien".

1981: Dr. Barbara Lau, Abteilung für Experimentelle Hämatologie der Gesellschaft für Strahlen- und Umweltforschung $\mathrm{mbH}$, München:

„Differenzierungsvermögen und Proliferation von normalen und malignen Zellen lymphatischen Ursprungs" (ein Auszug dieser Arbeit ist nachfolgend in diesem Heft abgedruckt). 


\section{Zur Verfügung stehende Mittel}

In den Jahren 1972-1982 sind der Stiftung über 3 Mill. DM zugeflossen. Jahr für Jahr gab Walter Reiners hohe Beträge, und auch die Familie hat bis in die jüngste Zeit durch weitere Zustiftungen das Vermögen der Stiftung weiter aufgefüllt. Im Verlaufe der Jahre haben sich in einer sehr dankenswerten Weise auch noch zahlreiche andere Personen den Uberlegungen angeschlossen, durch Zurverfügungstellung finanzieller Mittel die Leukämieforschung nach eigenem Vermögen zu unterstützen. Durch kleinere und größere Spendenbeträge von Dritten ist die Stiftung um DM 260 000,- bisher bereichert worden. Somit hält die Kind-Philipp-Stiftung den Charakter einer offenen Stiftung, offen für jedermann, der sich an einer Förderung der Leukämieforschung durch finanzielle Zuwendungen beteiligen will. Die Leukämie ist eine Krankheit, die jedermann und alle Familien bedroht. Nur wenige können als Wissen. schaftler direkt den Kampf gegen diese Krankheit aufnehmen. Durch finanzielle Zuwendungen kann die Forschung gegen die Leukämie ein allgemeines Anliegen werden. Ende Oktober 1982 betrug das Vermögen der Stiftung etwas über 1,4 Mill. DM.

\section{Dank an den Beirat}

Ein Rückblick auf die 10 Jahre erfolgreiche Kind-PhilippStiftung gibt Anlaß zu großer Dankbarkeit. Die Stiftung hat Walter Reiners nicht nur die Idee und das wirtschaftliche Fundament zu danken. Tatkräftig und drängend hat er sich als Vorsitzender des Beirates in den Sitzungen nicht nur an der Formulierung einer brauchbaren Förderungsstrategie beteiligt, sondern er ist immer wieder auch in die wissenschaftliche Offentlichkeit der Arbeitsgemeinschaft für Leukämieforschung mitgegangen, wenn es galt, neue Preisträger zu ehren und die Träger der klinischen und theoretischen Forschung kennenzulernen. Unvergeßlich ist er für viele, wie er mit jedem redete und reden konnte, fur jeden ein persönliches Wort hatte, wie er aufmerksam zuhörte und trotz seiner wissenschaftlichen Laiensituation den Problemkern der Leukämieforschung mitdenkend und hinterfragend erfassen konnte.

Er ist am 28. Januar $1980 \mathrm{im}$ 72. Lebensjahr verstorben, er ruht an der Seite seines jüngsten Sohnes Philipp. Für die Zukunft seiner Stiftung hat er gesorgt. In seinem Sinne und mit seinen Tendenzen wird weitergearbeitet. Den Vorsitz des Beirates wollte er in die Hände des seinen Sohn behandelnden Arztes gelegt wissen. Als Vertreter der Familie Reiners ist auf seinen Wunsch sein ältester Sohn Jan Reiners in den Beirat eingetreten; er bringt in seiner Person viele Eigenschaften seines Vaters mit.

Besonderer Dank gebührt den Generalsekretären des Stifterverbandes für die Deutsche Wissenschaft, in den ersten Jahren Thorwald Risler, seit 1979 Dr. Horst Niemeyer. Es ist ein wahres Glück für eine Stiftung dieser Art, die ganz auf ideeller Grundlage gegründet ist, wenn sie für alle notwendigen administrativen Belange sich auf die Erfahrung eines solchen Apparates stützen kann, seien es Fragen der finanziellen Bilanzierung, der fruchtbaren Geldpolitik zur Erhaltung des Vermögens, Fragen der steuerlichen Beratung oder auch Fragen der Wissenschaftsförderung in einem ganz allgemeinen Sinne. Von den zahlreichen Mitarbeitern
Mitglieder des Beirates der Kind-Philipp-Stiftung

Vorsitzender:

Dr.-Ing. Walter Reiners $\dagger$

seit 1980 Prof. Dr. Michael Hertl

Beiratsmitglieder:

Prof. Dr. Michael Hertl

Chefarzt der Kinderklinik des Krankenhauses

Neuwerk, Mönchengladbach

der Vorsitzende der Deutschen Arbeitsgemeinschaft für Leukämie-Forschung und -Behandlung im Kindesalter:

Prof. Dr. Günter Landbeck

Universitäts-Kinderklinik Hamburg

Direktor der Abteilung für Hämatologie und Onkologie

seit 1980 in seiner Nachfolge:

Prof. Dr. Fritz Lampert

Leiter der Universitäts-Kinder-Poliklinik Gießen

der Generalsekretär des Stifterverbandes für die Deutsche Wissenschaft:

\section{Thorwald Risler}

in seiner Nachfolge seit 1979:

Dr. Horst Niemeyer

ein Vertreter der Deutschen Forschungsgemeinschaft:

Dr. Fritz W. Fischer

in seiner Nachfolge seit 1980:

Prof. Dr. Dietrich Niethammer

Universitäts-Kinderklinik Tübingen

Direktor der Abteilung für pädiatrische Hämatologie

Jan Reiners

Persönlich haftender Gesellschafter

W. Schlafhorst \& Co., Mönchengladbach

seit 1980 als Vertreter der Stifterfamilie

Förderungsanträge sind unter dem Stichwort „KindPhilipp-Stiftung " $z u$ richten an den

Stifterverband für die Deutsche Wissenschaft, 4300 Essen 1, Postfach 230360, Brucker Holt 5660, Tel. 0201/711051

Auch für Zustiftungen zur gemeinnützigen-KindPhilipp-Stiftung gibt der Stifterverband die erwünschten Auskünfte.

Spenden an die Kind-Philipp-Stiftung können überwiesen werden an:

Gemeinnützige Verwaltungsgesellschaft für Wissenschaftspflege $\mathrm{mbH}$

(Verwaltungsgesellschaft des Stifterverbandes)

Sonderkonto

"Kind-Philipp-Stiftung für Leukämieforschung":

10007103 (BLZ 370302 00) beim Bankhaus

Sal. Oppenheim jr. \& Cie., Köln 
des Stifterverbandes haben vor allem Dr. Keller und Peter Beck sich für die Kind-Philipp-Stiftung besonders eingesetzt.

Die wichtigen praktischen Impulse für die Bewertung der eingereichten Anträge kamen innerhalb des Beirates natürlich in erster Linie von den wissenschaftlich-tätigen Mitgliedern. Ein Mitglied des Beirates ist der jeweilige Vorsitzende der Deutschen Arbeitsgemeinschaft für Leukämieforschung und -Behandlung im Kindesalter e.V., es war zunächst Prof. Landbeck, es ist nun in seiner Nachfolge Prof. Lampert. Ferner hat von Anfang an die Deutsche Forschungsgemeinschaft einen Vertreter in den Beirat gesandt, in den ersten Jahren Dr. Fritz W. Fischer, in seiner Nachfolge Prof. Niethammer.

Es fallt schwer, den Dank in einer angemessenen Form auszudrücken. Alle Mitglieder des Beirates haben viel Zeit geopfert, um eingereichte Anträge auf ihre wissenschaftliche Begründung und die Notwendigkeit des finanziellen Aufwandes zu prüfen, zunächst telefonisch zu diskutieren, schriftlich vorläufig zu beurteilen und dann in den regelmäBigen Beiratssitzungen endgültig zu entscheiden.

\section{Ausblick}

Auch in der Zukunft wird die Leukämieforschung Stiftungen in der Art der Kind-Philipp-Stiftung brauchen, will sie weiterkommen zum endgültig befriedigenden Ziel, die Grundlagen der Leukämiekrankheit voll zu erfassen und den Weg zur Heilung aller erkrankten Kinder und Erwachsenen ganz zu finden. Mit Blick auf die finanzielle Ausstattung der Kind-Philipp-Stiftung müssen wir hoffen, daß die wirtschaftliche Lage der Industrie es erlaubt, das Vermögen der Stiftung immer wieder aufzufüllen und daß weiterhin auch noch andere Personen die Möglichkeiten zur Zustiftung als Chance empfinden, einen eigenen Beitrag zur Wissenschaftsförderung zu leisten.

Soviel das heutige Therapiekonzept an langen Uberlebenszeiten und an Heilungen schon gebracht hat, so ist kaum zu glauben, daß auf dem heute beschrittenen Therapieweg durch Zytostase das Ziel einer Heilung aller Leukämiekranken gelegen sein wird. Auch in den Therapieverfahren von Pinkel und Riehm, so wertvoll und erfolgreich sie erscheinen, ist kein grundsätzlich neuer Weg gefunden, sondern der alte nur konsequent zu Ende gegangen worden. So sehr dankbar man heute für diese Therapiekonzepte sein will und sein muß, so kann doch in ihnen auch ein Hemmnis fur den weiteren therapeutischen Fortschritt, der noch darüber hinausgehen soll und muß, gelegen sein. Welcher verantwortungsbewußte Arzt könnte sich bei solchen Erfolgszahlen heute zu einem anderen Therapieschema entschließen und ein anderes Verfahren vor sich selbst und seinen Patienten verantworten? Im Grunde ist auch keine neue erfolgversprechende Therapietheorie heute in Sicht. Es bedarf somit unbedingt neuer Einsichten in die Zytobiologie der Leukämie, um daraus zu tragfähigen neuen, anderen Therapiekonzepten zu kommen. Ihre Erprobung wird ein Wagnis sein, das am Maßstab der Erfolgsstatistiken von Pinkel und Riehm gemessen werden wird. Zunächst ist nach einer Zeit vorwiegend spekulativ verbesserter Therapiesysteme wieder eine Zeit stiller Grundlagenforschung vonnöten.

Neue Erkenntnisse sind natürlich in erster Linie abhängig von gedankenreichen aufopfernd-tätigen Forschern. Sie sind aber gerade heute auch abhängig von materieller Hilfe für eine moderne Labortechnik und den Einsatz von medizinisch-technischen Mitarbeitern.

Diesen Gedanken wird sich die Kind-Philipp-Stiftung weiterhin verpflichtet wissen.

\section{Literatur:}

Hertl, M.: Dr. Walter Reinerst. Ergebnisse der Pädiatrischen Onkologie 4, S. 2-4 (1980); Enke-Verlag Stuttgart

Kind-Philipp-Expertentagung 1981. Ergebnisse der Pädiatrischen Onkologie 6, S. 281-282 (1982); Enke-Verlag Stuttgart 